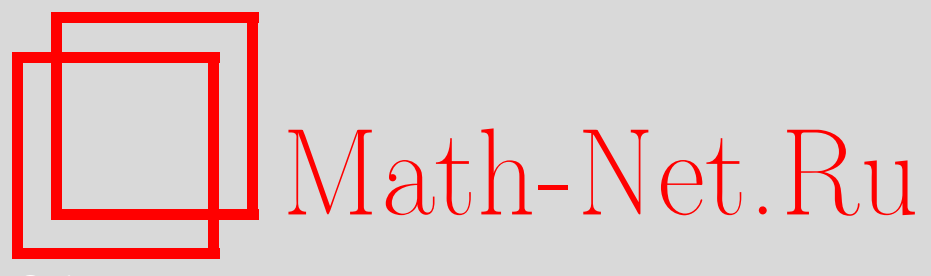

М. Н. Подоксенов, Нормальная подгруппа транзитивной группы конформных преобразований, имеющая орбиту коразмерности $k>1$, несущественна, Матем. заметки, 2007, том 82, выпуск 2, 317-320

DOI: https://doi.org/10.4213/mzm3786

Использование Общероссийского математического портала Math-Net.Ru подразумевает, что вы прочитали и согласны с пользовательским соглашением http://www . mathnet.ru/rus/agreement

Параметры загрузки:

IP: 3.80 .253 .173

26 апреля 2023 г., $11: 13: 17$

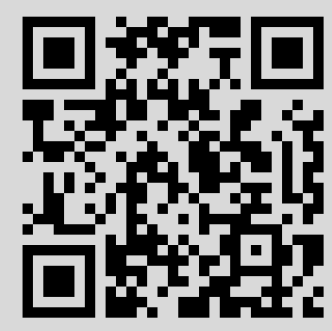




\section{Нормальная подгруппа транзитивной группы конформных преобразований, имеющая орбиту коразмерности $k>1$, несущественна}

\section{М. Н. Подоксенов}

Группа $G$ конформных преобразований риманова или псевдориманова многообразия $(M, g)$ называется несущественной, если существует такая метрика $\widetilde{g}$ на $M$, конформно эквивалентная $g$, что $G$ будет группой изометрий многообразия $(M, \widetilde{g})$, и существенной в противном случае.

Алексеевский [1] доказал, что риманово многообразие, обладающее связной существенной группой конформных преобразований, конформно эквивалентно сфере или евклидову пространству. Существует много примеров псевдоримановых многообразий с существенной группой конформных преобразований и полная их классификация до сих пор не получена. Лоренцевы многообразия, обладающие однопараметрической группой гомотетий, были описаны Алексеевским [2]. Некоторые частные результаты, описывающие топологическую структуру и метрику рассматриваемых многообразий, были получены автором [3], [4]. В работах [5]-[7] лишь доказывалось, что при определенных предположениях группы конформных преобразований являются либо несущественными, либо являются группами гомотетий данной метрики или конформно эквивалентной ей метрики.

В работе [7] автором было доказано, что нормальная подгруппа транзитивной группы конформных преобразований, обладающая неизотропной орбитой, несущественна. В настоящей работе мы отбросим требование неизотропности орбиты, но при этом необходимо будет установить ограничение на ее размерность. Список работ, в которых исследуются четырехмерные лоренцевы многообразия, допускающие инфинитезимальные группы гомотетий, можно найти в [2]. В настоящей статье будет доказана следующая

Теорема. Пусть $(M, g)$ - риманово или лоренцево многообразие, $G$ - группа Ли конформных преобразований, действующая транзитивно на нем, $H$ - ее нормальная подгруппа. Предположим, что для некоторого $p \in M$ орбита Нр замкнута и имеет коразмерность $k>1$, а стачионарная подгруппа $H_{p}$ свлзна. Тогда $H$ - несущественная группа конформных преобразований.

Следующий пример показывает, что в случае $k=1$ данная теорема для лоренцевых многообразий не имеет места.

Пусть $M$ - двумерное пространство Минковского с координатами $(u, v)$ и метрикой $d s=d u d v$. Пусть $\Phi=\left\{\varphi_{t}\right\}$ - однопараметрическая группа преобразований, действующая по формуле

$$
\varphi_{t}(u, v)=\left(e^{2 t} u, v\right) .
$$

Пусть $\Psi=\left\{\psi_{t}\right\}$ и $\Theta=\left\{\theta_{t}\right\}$ - группы переносов: $\psi_{t}(u, v)=(u+t, v)$ и $\theta_{t}(u, v)=(u, v+t)$. Нетрудно убедиться, что группа $\Theta$ коммутирует с $\Phi$ и с $\Psi$. Пусть $H$ - группа преобразований, порожденная группами $\Phi$ и $\Psi$. Тогда $H$ будет группой гомотетий, и причем, существенной. Пусть $G$ - группа преобразований, порожденная группами $H$ и $\Theta$. Тогда $G$ является группой конформных преобразований, и $H$ является нормальной подгруппой в $G$. При этом все орбиты группы $H$ изотропны и имеют коразмерность 1 . Нетрудно построить аналогичный пример и для пространства Минковского большей размерности.

ДоказАТЕЛЬСтво теоремы. Если $\operatorname{dim} H p=0$, то каждая орбита группы $H$ состоит только из одной точки и все они должны быть инвариантны относительно действия группы $H$. Значит, $H$ состоит только из тождественных преобразований и поэтому несущественна. 
Пусть теперь $\operatorname{dim} H p \neq 0$. Если $H p$ - неизотропное подмногообразие в $(M, g)$, то данная теорема доказана в [7].

Пусть $(M, g)$ - лоренцево многообразие, $n=\operatorname{dim} M$, а $H p$ - изотропное подмногообразие в $M$. Поскольку $H$ является нормальной подгруппой, то любой элемент из $G$ переводит орбиты группы $H$ в орбиты группы $H$. Поэтому все орбиты $H q, q \in M$, замкнуты, имеют одинаковую размерность и на всех орбитах индуцируется полуриманово скалярное произведение, а стационарная подгруппа $H_{q}$ связна для любого $q \in M$.

Пусть $p \in M-$ произвольная точка, $N=H p$ - ее орбита группы $H$. Пусть $h \in H_{p}$ произвольный элемент, который можно соединить с единицей группы с помощью однопараметрической подгруппы $\Phi=\left\{\varphi_{t}\right\} \subset H_{p}$, и пусть $h=\varphi_{t_{1}}$. Тогда согласно [2] преобразование $\left(\varphi_{t *}\right)_{p}: T_{p} M \rightarrow T_{p} M$ задается одной из следующих матриц в соответствующем базисе $\left\{e_{1}, e_{2}, \ldots, e_{n}\right\} \subset T_{p} M$ :

$$
A(t)=e^{\mu t}\left(\begin{array}{ccc}
e^{t \nu} & & \\
& S(t) & \\
& & e^{-t \nu}
\end{array}\right), \quad B(t)=e^{\mu t}\left(\begin{array}{ccc|c}
1 & 0 & 0 & \\
t & 1 & 0 & \\
t^{2} / 2 & t & 1 & \\
\hline & & & S_{1}(t)
\end{array}\right),
$$

где $S(t) \in O(n-2), S_{1}(t) \in O(n-3)$. При этом матрицы скалярных произведений данных базисов имеют вид

$$
\left(\begin{array}{lll} 
& & 1 \\
& E_{1} & \\
1 & &
\end{array}\right), \quad\left(\begin{array}{lll|l}
0 & 0 & 1 & \\
0 & 1 & 0 & \\
1 & 0 & 1 & \\
\hline & & E_{2}
\end{array}\right)
$$

где $E_{1}$ и $E_{2}$ - единичные матрицы порядков $n-2$ и $n-3$ соответственно.

Предположим сначала, что $\left(\varphi_{t *}\right)_{p}$ задается матрицей $A(t)$. Мы докажем, что $\mu=0$, и тем самым покажем, что $\left(h_{*}\right)_{p}$ изометрично.

Подпространство $T_{p} N$ является изотропным в $T_{p} M$, и оно инвариантно относительно $\left(\varphi_{t *}\right)_{p}$. Это подпространство содержит единственное изотропное направление $I_{2}$, и это направление тоже должно быть инвариантно относительно действия $\left(\varphi_{t *}\right)_{p}$. Значит, $I_{2}=$ $R e_{1}$ или $I_{2}=R e_{n}$. Будем считать, что $I_{2}=R e_{n}$, и пусть $I_{1}=R e_{1}$.

Обозначим $L=\left(I_{1} \oplus I_{2}\right)^{\perp}-(n-2)$-мерное подпространство в $T_{p} M$. На нем индуцируется евклидово скалярное произведение. Поскольку $\operatorname{codim} T_{p} N=k>1$, то заведомо можно утверждать, что $L \not \subset T_{p} N$. Пусть $L^{\prime \prime}=T_{p} N \cap L$, а $L^{\prime}$ - его ортогональное дополнение в $L$. Тогда оба подпространства $L^{\prime}$ и $L^{\prime \prime}$ инвариантны относительно действия $\left(\varphi_{t *}\right)_{p}$, и это преобразование действует на $L^{\prime}$ и $L^{\prime \prime}$ как растягивающее или сжимающее, если $\mu \neq 0$.

Пусть $G^{\prime}$ - множество всех однопараметрических подгрупп, касательный вектор к которым принадлежит $L^{\prime}$, а $\mathscr{L}$ - объединение орбит всех таких подгрупп. В силу гладкости действия группы $G$ на $M$ множество $\mathscr{L}$ будет гладким подмногообразием в $M$. Используя замкнутость орбиты $N$, можно доказать, что существует такая окрестность $U$ точки $p$ в $\mathscr{L}$, что в $U$ нет других точек из $N$. В силу гладкости действия группы $G$ на $M$ найдется такая окрестность $U_{1}$ точки $p$ в $U$, что $T_{q}(H q) \cap T_{q} \mathscr{L}=\{\vec{o}\}$ выполнено для каждого $q \in U_{1}$.

Введем на $U_{1}$ вспомогательную риманову метрику, и пусть $\rho$ - функция расстояния этой метрики. Пусть $\varepsilon>0$ таково, что открытый шар $B(p, \varepsilon) \subset U_{1}$ предкомпактен, и пусть $U_{2}$ - его компактное замыкание. В силу замкнутости каждой орбиты группы $H$ и гладкости действия $G$ у каждой точки $q \in U_{2}$ существует шаровая $\delta$-окрестность, в которой нет других точек орбиты $H q$, кроме $q$. Далее легко доказать, что можно выбрать $\delta_{o}$ единое для всех $q \in U_{2}$. Пусть теперь $V=B\left(p, \delta_{o}\right) \in U_{2}$.

Пусть $q \in V$ - произвольная точка. Тогда существуют однопараметрическая подгруппа $\Psi=\left\{\psi_{\tau}\right\} \in G^{\prime}$ и число $\tau_{o}$ такие, что $q=\psi_{\tau_{o}} p$. Тогда $\psi_{\tau_{o}}(N)=H q$. Выберем $n \in Z$ достаточно большим, чтобы для $t_{o}=t_{1} / n$ выполнялось $\varphi_{t_{o}} q \in V$ и $\varphi_{-t_{o}} q \in V$. 
Пусть $h_{o}=\varphi_{t_{o}}$. Пусть $\theta_{\tau}=h_{o}^{-1} \psi_{\tau} h_{o}$. Тогда $\Theta=\left\{\theta_{\tau}\right\}$ тоже будет однопараметрической подгруппой и $\Theta \in G^{\prime}$, поскольку $L^{\prime}$ инвариантно относительно $\left(h_{o *}\right)_{p}$. Имеем $\theta_{\tau_{o}} p=h_{o}^{-1} q \in H q$, и в то же время $\theta_{\tau_{o}} p \in V$. Но $H q \cap V=\{q\}$, следовательно, $h_{o}^{-1} q=q$ и $h_{o} q=q$. Тогда $h q=\left(h_{o}\right)^{n} q=q$. Поскольку $q$ была произвольной точкой из $V$, то $h$ действует на $V$ тождественно и $\mu=0$. Значит, $\left(h_{*}\right)_{p}$ изометрично.

Если теперь $h^{\prime} \in H_{p}$ - произвольный элемент, который нельзя соединить с единицей с помощью однопараметрической подгруппы, то $h^{\prime}=h_{m} \cdot h_{m-1} \cdots h_{1}$, где каждый из $h_{i}$, $i=1, \ldots, m$, можно соединить с единицей. Поэтому $\left(h_{*}^{\prime}\right)_{p}$ тоже изометрично для любого $h^{\prime} \in H_{p}$.

Подпространство $I_{2}$ инвариантно относительно $\left(\varphi_{t *}\right)_{p}$. Поэтому, применяя к $I_{2}$ дословно такие же рассуждения, как и к $L^{\prime}$, мы получим, что $\left(\varphi_{t *}\right)_{p}$ действует на $I_{2}$ тождественно. Поэтому $\nu=0$. Значит, $\left(\varphi_{t *}\right)_{p}$ действует тождественно на подпространстве $P=L^{\prime} \oplus I_{2} \subset T_{p} M$. При этом, $P \oplus T_{p} N=T_{p} M$.

Пусть $\mathscr{P}$ - объединение орбит всех однопараметрических подгрупп, касательные векторы к которым принадлежат $P$, а $G^{\prime \prime}$ - совокупность всех таких подгрупп. В силу гладкости действия $G$ на $M$ множество $\mathscr{P}$ представляет собой гладкое подмногообразие в $M$. Так же, как мы построили $V \subset L$, мы можем построить предкомпактную окрестность $W$ точки $p$ в $P$, обладающую следующими свойствами: для каждой точки $q \in W$ орбита $H q$ пересекает $W$ только в точке $q$ и при этом подпространства $T_{q} H q$ и $T_{q} W$ трансверсальны в $T_{q} M$. Тогда множество $W$ представляет собой срез или сляйс (терминология Алексеевского [1; с. 284]), т.е. оно обладает следующими свойствами:

1) $H W$ содержит окрестность точки $p$;

2) если для $x, y \in W$ выполнено $a x=y, a \in H$, то $a \in H_{p}$.

Заметим, что $W$ инвариантно относительно действия однопараметрической подгруппы $\Phi$, но оно может быть не инвариантно относительно подгруппы $H_{p}$ в целом.

Пусть $\widetilde{W}=\bigcup_{q \in W} H q$ - трубчатая окрестность точки $p$, содержащая $W$. Тогда $H \widetilde{W}=\widetilde{W}$. Определим на $\widetilde{W}$ новую метрику $\widetilde{g}$ следующим образом. Пусть $q \in W$, а $r \in H q$. Пусть $a \in H$ - такой элемент, что $r=a q$. Пусть $f_{a}(q)$ - коэффициент гомотетии преобразования $a$ в точке $q$. Тогда положим $\widetilde{g}=f_{a}(q) g_{r}$. Если $b \in H$ - другой элемент такой, что $r=b q$, то $b=a h$, для некоторого $h \in H_{q}$. В силу того, что всякое преобразование $h \in H_{q}$ изометрично в точке $q$, то $f_{a}(q)=f_{b}(q)$. Поэтому определение $\widetilde{g}_{r}$ корректно. А гладкость действия $H$ на $M$ приводит к тому, что новая метрика на $\widetilde{W}$ тоже будет гладкой.

Пусть $M^{\prime}=H \backslash M$ - пространство орбит группы $H$, и пусть $\pi: M \rightarrow M^{\prime}$ - естественная проекция. Используя существование среза для любой точки $p \in M$, легко доказать, что $M^{\prime}$ хаусдорфово, локально компактно и паракомпактно (и нормально); см. [1; с. 285]. Для каждого $p \in M$ построим свой срез $W_{p}$. Тогда множества $\bar{W}_{p}=\pi\left(\widetilde{W}_{p}\right)$ образуют покрытие пространства $M^{\prime}$, и можно доказать, что каждое из этих множеств предкомпактно. Впишем в это покрытие локально конечное покрытие $\left\{\bar{W}_{\alpha}\right\}$ (для удобства обозначений мы используем ту же букву) и построим разложение единицы $\left\{\omega_{\alpha}\right\}$ на $M^{\prime}$, подчиненное этому покрытию. Оно естественным образом определяет $H$-инвариантное разложение единицы $\left\{\widetilde{\omega}_{\alpha}\right\}$ на $M$.

Пусть $\widetilde{W}_{i}$ и $\widetilde{W}_{j}$ - две пересекающиеся трубчатые окрестности, а $\widetilde{W}_{i j}=\widetilde{W}_{i} \cap \widetilde{W}_{j}$. Пусть $\widetilde{g}^{i}$ и $\widetilde{g}^{j}$ - две метрики, определенные на $\widetilde{W}_{i}$ и $\widetilde{W}_{j}$ соответственно согласно описанному выше правилу. Обе эти метрики конформно эквивалентны $g$, а значит, они эквивалентны друг другу: их компоненты пропорциональны. Поэтому мы можем легко определить метрический тензор $\widetilde{g}^{i j}$ на $\widetilde{W}_{i j}: g^{i j}=\omega_{i} g^{i}+\omega_{j} g^{j}$ (без суммирования по $i$ и $j$ ). Метрика $g^{i j}$ конформно эквивалентна каждой из рассмотренных выше метрик. Поскольку группа $H$ действует на $\widetilde{W}_{i j}$ изометрично относительно $g^{i}$ и относительно $g^{j}$, то она действует изометрично и относительно $g^{i j}$.

Нетрудно доказать по индукции, что метрика $\widetilde{g}=\sum_{\alpha} \omega_{\alpha} \widetilde{g}^{\alpha}$, определенная на $M$, конформно эквивалентна $g$ и группа $H$ действует на $M$ изометрично относительно $\widetilde{g}$. 
Пусть теперь $\left(\varphi_{t *}\right)_{p}$ определяется матрицей $B(t)$. Тогда единственным изотропным направлением, инвариантным относительно действия $\left(\varphi_{t *}\right)_{p}$, является $I_{3}=R e_{3}$. Поскольку изотропное направление в $T_{p} N$ должно быть инвариантно, то $e_{3} \in T_{p} N$. Векторы $e_{k+2}, \ldots, e_{n}$ можно выбрать так, чтобы они лежали в $T_{p} N$, а $e_{2}, e_{4}, \ldots, e_{k+1}$ - чтобы были ортогональны $T_{p} N$ (изотропный вектор $e_{1}$ хоть и не лежит в $T_{p} N$, но ему не ортогонален).

Пусть $\Psi=\left\{\psi_{\tau}\right\} \subset G$ - однопараметрическая подгруппа, орбита $\Psi p$ которой имеет начальный вектор $e_{1}$. Введем в некоторой окрестности $U$ точки $p$ в $M$ гладкую систему координат так, чтобы $\Psi p \cap U$ задавалась системой уравнений $x_{2}=\cdots=x_{n}=0$ (это возможно в силу [8; лемма 1 , с. 22$])$, а $T_{p} N$ имела в качестве базиса $\left\{e_{3}=\partial / \partial x^{3}, e_{k+2}=\right.$ $\left.\partial / \partial x^{k+2}, \ldots, e_{n}=\partial / \partial x^{n}\right\}$ (если последнее не выполняется, то можем совершить линейную замену координат). При необходимости можем совершить гладкую замену координат, изменив только $x^{1}$, так, чтобы $\psi_{\tau} p$ имела координаты $(\tau, 0, \ldots, 0)$. Пусть $\tau$ достаточно мало и $q=\psi_{\tau} p$ - близкая к $p$ точка. Тогда в силу гладкости действия группы $G$ на $M$ касательное пространство к $H q=\psi_{\tau}(N)$ в точке $q$ будет иметь в качестве базисных векторы, отличающиеся от $\left\{\partial / \partial x^{3}, \partial / \partial x^{k+2}, \ldots, \partial / \partial x^{n}\right\}$ на векторные величины порядка не выше $\tau$. Пусть $t$ также мало. Разложим координатные функции, представляющие отображение $\varphi_{t}$, в ряд Тейлора в окрестности точки $p$ :

$$
\varphi_{t}^{i}(x)=b_{t}^{i}(t) x^{j}+(1 / 2) d_{j k}^{i}(t) x^{j} x^{k}+o_{t}^{i}\left(\left(x^{1}\right)^{2}+\cdots+\left(x^{n}\right)^{2}\right), \quad i=1, \ldots, n,
$$

где $o_{t}^{i}(x)$ - бесконечно малые величины по сравнению с $x$. Тогда применительно к точке $q$ имеем

$$
\varphi_{t}^{i}(q)=b_{1}^{i}(t) \tau+(1 / 2) d_{11}^{i}(t) \tau^{2}+o_{t}^{i}\left(\tau^{2}\right), \quad i=1, \ldots, n .
$$

Значит, $\varphi_{t}(q)$ имеет координаты

$$
\left(e^{\mu t} \tau+O_{t}^{1}\left(\tau^{2}\right), e^{\mu t} t \tau+O_{t}^{2}\left(\tau^{2}\right),(1 / 2) e^{\mu t} t^{2} \tau+O_{t}^{2}\left(\tau^{2}\right), O_{t}^{4}\left(\tau^{2}\right), \ldots, O_{t}^{n}\left(\tau^{2}\right)\right),
$$

где $O_{t}^{i}\left(\tau^{2}\right), i=1, \ldots, n,-$ величины порядка не ниже, чем $\tau^{2}$. Дифференцируя при $t=0$, получаем, что касательный вектор к траектории $c(t)=\varphi_{t} q$ имеет в точке $q$ координаты, отличающиеся от $(\mu \tau, \tau, 0, \ldots, 0)$ на величину порядка не ниже, чем $\tau^{2}$. Следовательно, при малых $\tau$ вектор $\vec{c}^{\prime}(0)$ не может содержаться в $T_{q}(H q)$, а значит, траектория $c(t)$ точки $q$ не может содержаться в $H q$. Но $\Phi \subset H_{p}$, поэтому $c(t) \subset H q$. Полученное противоречие означает, что $\left(\varphi_{t *}\right)_{p}$ не может задаваться матрицей $B(t)$.

\section{СПИСОК ЦИТИРОВАННОЙ ЛИТЕРАТУРЫ}

[1] Д. В. Алексеевский, Матем. сб., 89:2 (1972), 280-296. [2] D. Alekseevski, Ann. Global Anal. Geom., 3:1 (1985), 59-84. [3] М. Н. Подоксенов, Сиб. матем. журн., 30:5 (1989), 135-137. [4] М. Н. Подоксенов, Сиб. матем. журн., 34:2 (1993), 146-153. [5] C. Barbance, C. R. Acad. Sci. Paris. Ser. A, 291:5 (1980), 347-350. [6] M. N. Podoksenov, Differential Geometry and Applications, Proceedings of the 6th international conference (Brno, Czech Republic, August 28-September 1, 1995), 1996, 61-64. [7] М. Н. Подоксенов, Вестн. Вuтебского гос. ун-та, 2001, № 4(22), 75-77. [8] Д. Громол, В. Клингенберг, В. Мейер, Риманова геометрия в иелом, Мир, М., 1971.

М. Н. Подоксенов

Витебский государственный университет, Беларусь E-mail: geom@vsu.by
Поступило

16.07.2004

Исправленный вариант 22.02.2007 